Published in Swiss journal of psychology 2020, vol. 79 no. 3-4,

pp. 101-112, which should be cited to refer to this work. DOI:

\title{
How musicality, cognition and sensorimotor skills relate in
}

\section{musically untrained children}

Clara E. James ${ }^{1,2}$, Sascha Zuber ${ }^{2}$, Elise Dupuis-Lozeron ${ }^{3}$, Laura Abdili ${ }^{2}$, Diane Gervaise ${ }^{2}$ \& Matthias Kliegel $2,4,5$

${ }^{1}$ Geneva School of Health Sciences, HES-SO, University of Applied Sciences and Arts

Western Switzerland, Geneva, Switzerland

${ }^{2}$ Department of Psychology, University of Geneva, Geneva, Switzerland

${ }^{3}$ Division of Clinical Epidemiology, University Hospital of Geneva, Geneva, Switzerland

${ }^{4}$ Center for the Interdisciplinary Study of Gerontology and Vulnerability, University of

Geneva, Switzerland.

${ }^{5}$ Swiss National Center of Competences in Research LIVES-Overcoming vulnerability: Life

Course Perspectives, Lausanne and Geneva, Switzerland.

\section{Corresponding author}

Clara E. James

Geneva School of Health Sciences HES-SO

Avenue de Champel 47

1206 Geneva, Switzerland

clara.james@hesge.ch 


\begin{abstract}
Whereas a growing corpus of research has investigated the impact of music practice on several domains of cognition, studies on the relationships between musicality and other abilities and skills in musically untrained children are scarce. The present study examined the associations between musicality, cognition and sensorimotor skills in 69 musically untrained primary school children of around 10 years of age, using a test battery of musical, cognitive and sensorimotor abilities. We analyzed the results using non-parametric correlations and an exploratory factor analysis.

It was our anticipation that basic cognitive resources (short-term and working memory, attention, processing speed) would relate to both higher-order cognition and musicality.

Results indicated that, in musically untrained children, the interconnections between musical and cognitive abilities restrain to auditory short-term and working memory. Direct associations between musicality and higher-order cognitive processes did not occur.

An interesting secondary finding comprised associations between sensorimotor function, as measured by the Purdue Pegboard test, and higher-order cognition. Specifically, we found an association between bimanual coordination of fine finger dexterity, and matrix reasoning. This outcome suggests that higher-order cognitive function benefits from an efficient mastering of procedural aspects of sensorimotor skills.
\end{abstract}

\title{
Keywords:
}

primary school children, non-musicians, musicality, cognition, sensorimotor skills 


\section{Introduction}

The present study explored the links between musicality, cognition and sensorimotor abilities in 69 musically untrained primary school children of around 10 years of age using an ad-hoc test battery of musical, cognitive and procedural abilities.

The human brain seems prewired for music processing, both concerning pitch (Perani et al., 2010) and rhythm (Winkler et al., 2009). Neonates responded in a specific right-sided brain network to well-structured dance music but not to scrambled versions of the same stimuli (Perani et al., 2010). Other newborns emitted mismatch-like responses to unexpected omissions of the strong beat within the measure (Winkler et al., 2009). These observations support the hypothesis, that universal aspects of musicality are innate and a basic ingredient of early perceptual and cognitive function and development (Hannon \& Trainor, 2007).

More specifically, in early infancy, musical hearing and ability are supposed essential to language acquisition: "Without the ability to hear musically, we would be unable to learn to speak" (Brandt et al., 2012, p. 327). Brandt and colleagues argue compellingly that natural language and music development -the latter without explicit training- mature along similar tracks, both following enculturation by mere exposure (Hannon \& Trainor, 2007). In 5- to 7year-old children, interrelationships between language and music skills occurred, particularly if similar processes were involved like syntax processing (Cohrdes et al., 2016). Apart from links to language skills, however, few empirical data exist regarding the associations between musicality and more general cognitive processing abilities in musically untrained children. An awareness of musical syntax incongruity in simple and complex music manifested from as early as 6 years of age in both musically naïve and musically trained primary school children (James, Dupuis-Lozeron, et al., 2012). Additionally, in an EEG (electroencephalography) study, 16 musically untrained school children of around ten years of age, better detected musical transgressions with increasing auditory working memory scores (James et al., 2015). 
Musical practice, i.e. actively playing an instrument or singing, covering a wide and diverse field of competences -ranging from sensorimotor to cognitive processing activities of the highest level- manifests as a driving force of cognitive and sensorimotor development. The impact of music practice on several domains of cognition, including language, is supported by a growing corpus of research (Anvari et al., 2002; Courey et al., 2012; Degé et al., 2011; James et al., 2020; Jaschke et al., 2018; Martins et al., 2018; Miendlarzewska \& Trost, 2013; Moreno et al., 2009, 2011; Rauscher et al., 1997; Rickard et al., 2012; Roden et al., 2012, 2014; Rose et al., 2015; Schellenberg, 2004, 2006; Seither-Preisler et al., 2014). Although some methodological weaknesses may be present in these studies, partially because of lack of consensus on definitions of musicality and of transfer (Jaschke et al., 2013), it seems that, when practicing musical skill specifically and actively over longer time periods, links between musical and cognitive abilities become gradually entangled and enhanced. This observation supposes that certain basic cognitive processing resources are shared between general and musical cognition.

Research on the relationships between musical cognition and other domains in naturally developing non-musician children is still scarce. One study reported that the discrimination of musical rhythm predicted grammar skills in 6-year-olds (Gordon et al., 2015).

Findings are diverse on the relationship between cognition and sensorimotor skill. Two studies showed that the development of fine motor skills before the age of four does not predict cognitive performance during school age (Piek et al., 2008; Wang et al., 2014). In young adults, motor timing and abstract reasoning positively correlated (Lorås et al., 2013). In children between sic and nine years of age, performance on a visuomotor precision task was positively related to measures of intelligence (Mous et al., 2017), and in preschoolers, fine motor skills positively influenced lexical processing (Suggate \& Stoeger, 2017). 
Data are essentially lacking concerning the relationship between musical abilities and sensorimotor skill in non-musician children.

We conducted our study in public primary schools in popular neighborhoods in the vicinity of Geneva, with children of varying ethnic and socio-economic backgrounds. We explored how naturally developed musicality, i.e. by means of mere exposition in daily life, thus implicit learning, related to other domains, such as verbal memory and learning, processing speed, mental flexibility, attention, auditory memory, matrix reasoning and sensorimotor skills. This battery was composed of tests measuring abilities implied in musical, cognitive and sensorimotor activities that might potentially be associated.

Because possessing good discriminatory skills for pitch and rhythm is not sufficient to evaluate musicality, we adopted Karma's definition of musical aptitude (Karma, 2007) as representing "auditory structuring", expressing the capacity to group individual notes into gestalts and to form expectancies. To that end, we used a musical test that goes beyond mere examining of good auditory discrimination, namely, Gordon's Advanced Measures of Music Audiation, involving comparing musical phrases.

We analyzed the results by means of simple relationships between the scores of all different tests and also within an exploratory factor analysis.

The current study examined the following assumptions. First, we hypothesized that musical abilities are related to basic cognitive resources, namely, short-term memory, working memory, attention, processing speed and mental flexibility (Bergman Nutley et al., 2014; James et al., 2015, 2020; Roden et al., 2014). Second, we assumed that cognition and sensorimotor function may be related, since first expressions of intelligence are sensorimotorrelated (Anderson et al., 2013) and simple but especially complex motor tasks demand good organization in time and space and thus some abstract reasoning (Lorås et al., 2013). Third, since musical experience is intrinsically related to sensorimotor behavior in non-musicians, 
especially for rhythm perception (Chen et al., 2008; Grahn \& Rowe, 2009; James, Michel, et al., 2012), musical performance in the rhythm perception domain and sensorimotor performance may correlate.

\section{Methods}

\section{Participants}

Sixty-nine primary schoolchildren participated in the study, with a mean age of 10.18 years $(\mathrm{SD}=0.31 ; 41$ girls). They all attended public schools in the Geneva canton, in Frenchspeaking Switzerland. The group contained children of varying ethnic and socioeconomic backgrounds. The children had almost finished their sixth year of elementary school, which in Switzerland lasts approximately from age four to twelve years (eight consecutive years). One of the schools prohibited collecting precise information on the education level and actual professional occupation of the parents for ethical reasons that we can therefore not report here. We requested that none of the children who participated in the study, followed regular or protocolled music practice outside the school curriculum. Seven children were lefthanded. The children and their caregivers signed an informed consent. The ethics commission of the Faculty of Psychology and Educational Sciences of the University of Geneva approved the protocol, in agreement with the ethical standards of the declaration of Helsinki.

\section{Procedure}

The experimenters, all Master's students at the Psychology Department of the University of Geneva, tested children individually. Testing took place within the schools, in specifically selected quiet rooms. First the experimenter explained the overall aim of the study: "You are going to participate in a study of the University of Geneva that investigates how children develop." They then encouraged the child to ask questions and emphasized that they may ask 
for a break at any time.

All tests listed below (Table 1) were administered in pseudorandomized order, though the word vocabulary test was always presented relatively early, as a delayed word recall required a 50min suspension.

Total testing time was less than $1 \mathrm{~h}$ and $30 \mathrm{~min}$, spread over approximately $2 \mathrm{~h}$, separated by breaks. Children enjoyed participating, and only a few complained about fatigue. They all received a small gift at the end of the session.

\section{INSERT TABLE 1 ABOUT HERE}

\section{Materials}

Table 1 lists all tests, measured variables, acronyms of the tests and involved abilities

\section{Music Audiation}

In order to assess general music processing, comprising the melodic and the rhythm domain, we administered the "Advanced Measures of Music Audiation" (AMMA; Gordon, 1989). This test does not require any prior musical knowledge or skills. It encompasses 30 trials consisting of pairs of musical melodies or phrases presented over headphones via the computer. The test can be applied to children from the seventh grade, without any prior musical training. For each pair the children judged whether the melodies were identical or different. If they considered the two melodies of the pair different, they had to express whether the difference was rhythmic or melodic. The test is constructed such that only one type of difference (melodic or rhythmic) may occur between the two melodies at a time. The experimenters noted the answers on an answer sheet. Because the scoring system penalizes errors, we encouraged the children not to respond if they were not sure what to answer. The answer sheet therefore contained four 
columns: identical - melodic difference - rhythmic difference - I don't know. After an initial explanation of the concepts of melody and rhythm, and before the actual test, three training trials that did not appear in the final test were presented and discussed with the children to assure their comprehension of the instructions. We computed a tonal and a rhythmic score, according to the AMMA manual (Gordon, 1989), which involves subtracting points for wrong answers for both scores. From these scores we inferred percentile rank scores according to the AMMA manual, which we used for the analyses.

We do not report the composite score, which is a simple addition of both the tonal and the rhythm score and does not supply any additional information.

\section{Matrix reasoning}

Music cognition implies some abstract reasoning, as musical phrases evolve over time according to musical syntax, like language (Patel, 2012). In order to assess children's abstract reasoning skills, we administered the Matrices subtest of the WISC-IV (Wechsler, 2003). We presented different sheets with series of three images (e.g., three skateboards) to the children, whose task was to find the image that correctly completed the series (e.g., another skateboard) among four distractors (e.g., other toys). To familiarize the children with the task, we began with three practice trials. For the actual tasks, the sheets progressively increased in difficulty. Children worked on the task until they answered four out of five consecutive sheets incorrectly. The final score of Matrix Reasoning (MR) represents the number of correctly answered sheets.

\section{Selective visual attention}

To assess children's selective visual attention, we administered the D2 Test of Attention (Brickenkamp \& Zillmer, 1998). For this task, we presented a sheet with 14 rows of 47 stimuli. 
Stimuli were the letters $\mathrm{d}$ or $\mathrm{p}$ accompanied by one or two apostrophes above and/or below the letter. The children's task was to cross out all the d's that were accompanied by exactly two apostrophes (i.e., two apostrophes above, two apostrophes below or one above and one below the d) without crossing out any of the distractors (d's accompanied by only one apostrophe and all p's). To familiarize participants with the task, children first performed a practice row of 22 trials. For the actual task, children started working on the first row and were asked to switch to the next row every $20 \mathrm{~s}$. The outcome measure (D2) provides the number of marked items minus the number of errors and omissions.

\section{Visual processing speed and mental flexibility}

To assess children's visual processing speed and mental flexibility, we applied the Children's Color Trails Test (CCTT) (Llorente, 2003), which consists of two subtests. For test 1 (CCTT1), children were presented a sheet with 15 circles containing the digits " 1 " to " 15 ." Their task was to connect the digits in increasing order. All circles with even digits were colored yellow, whereas the circles with odd numbers were colored pink. For test 2 (CCTT-2), the children had to perform the same task, except that for each digit (except for "1") two circles were depicted on the sheet, one colored yellow, the other colored pink. Thus, the children were instructed that, in addition to connecting the digits in increasing order, the colors of the circles would have to alternate for each digit (the pink "1" had to be connected with the yellow "2," which had to be connected to the pink " 3 ," etc.). The first subtest evaluates simple visual processing speed, the second one also mental flexibility. To familiarize the children with the tasks, we began tests 1 and 2 with an 8-digit practice sheet. We computed standard scores as outcomes for both subtests $(M=100 ; S D=15)$. For the analyses we used percentile scores. 


\section{Digit span forward and backward}

In order to assess a measure of auditory short-term memory and working memory, the children accomplished the "digit span" subtest of the Wechsler Intelligence Scale for Children Revised (Wechsler, 2005). During these tests, the children listened to recorded spoken series of digits with increasing length. We used prerecorded spoken series to assure a regular pace (1 s per digit) and identical pronunciation for all participants. The participants' task consisted of repeating the series orally, in the first task in direct, in the second in reverse order. Digit Span Forward (DSF) and Digit Span Backward (DSB) tasks assess distinct but interdependent cognitive functions (Grégoire, 2009). DSF evaluates essentially serial short-term auditory memory, whereas DSB evaluates the child's ability to manipulate verbal information while temporarily stored, thus principally working memory capacity. Two digits series (one for each task), progressively increasing in length and thus in difficulty, were presented. The children first performed the DSF (span size from 2 up to 9) then the DSB task (span size from 2 up to 8). They proceeded through the trials until they made two successive mistakes with the same number of digits, i.e., at the same level of difficulty. Each correct answer merits one point for a total of 16 points per task.

\section{Rey Auditory Verbal Learning Test}

The experimenters orally presented a list of 15 unrelated words to the children (Bean, 2011; Rey, 1964), repeated over five different trials. The task consisted of repeating as many correct words out of the list as possible after a short break of approx. $10 \mathrm{~s}$. For the first trial, the time limit for recollection was set at $1 \mathrm{~min}$, for trials $2-5$ to $1 \mathrm{~min}$ and $30 \mathrm{~s}$. The list was read aloud first every time. Trials $2-5$ are performed immediately after trial 1 . After a delay of approx.50 min, the child should once more cite as many words as possible from the list, but this time, without their oral presentation before. The scores correspond each time to the number of 
correctly repeated words. We used the following measures: (1) score trial 1 (Rey-1); (2) mean score trials 2-5 (Rey-2); (3) score delayed recall (Rey-3). Rey-1 represents principally verbal short-term memory (STM), Rey-2 verbal learning, and Rey-3 verbal long-term memory (LTM).

\section{Fine and gross sensorimotor skills}

We used the Purdue Pegboard (PP) test, administered according to the Lafayette manual (Lafayette, 1999), to evaluate manual fine and gross dexterity and bimanual coordination. The Pegboard contains 2 rows of 25 holes, oriented vertically. Two cups on top of the board contain pegs (diameter $1 \mathrm{~mm}$ ), collars and washers. After a series of practice trials, the children inserted as many pegs as possible in the holes in $30 \mathrm{~s}$, from top to bottom, using their right hand (PP$\mathrm{RH})$, then their left hand (PP-LH), and finally with both hands simultaneously (PP-BH). These tasks evaluate gross hand dexterity and bimanual coordination, respectively. Then the children performed an assembly task working with both hands, placing as many assemblies in the holes as possible within $1 \mathrm{~min}$ (PP-Ass). This subtask requires bimanual coordination in combination with fine finger dexterity. One assembly consisted of a peg, a collar, and two washers (four elements) to be placed into one hole in a specific order. Four scores were collected, corresponding to the number of pegs placed (PP-RH, PP-LH, PP-BH) and the number of correctly inserted elements placed (PP-Ass).

\section{Missing data}

We report a total of six missing entrees (69 children, 15 different measures). Common approaches like substituting missing data by the group mean, induces the risk of reducing statistical variability and study validity. In consequence, we imputed the missing values using 
the regularized iterative principal component analysis (PCA) algorithm developed by Josse et al. (2009), as implemented in the R package missMDA.

\section{Correlation matrix}

The root of our analyses consists of a simple correlation matrix using Spearman's nonparametric rho on ranks. We opted for a rank-based coefficient, as some outliers were present in our measures (see boxplots of all variables in supplementary Figure 1). As this is an exploratory study, we report all correlations with a $p$-value $<.05$ (uncorrected).

\section{Exploratory Factor Analysis}

We chose an exploratory factor analysis given that our hypotheses express relationships between our test measures that may share common underlying processing elements of which the structure is unknown (Child, 2006).

In order to determine an optimal number of factors for the Exploratory Factor Analysis, we performed 1) a Principal Component Analysis (PCA) and 2) a Parallel Factor Analysis (PFA) (Desjardins, 2018; Horn, 1965). We performed a PCA on the whole data set, using the R package FactoMineR (http://www.R-project.org). Before performing the PCA all data were first centered $(M=0.0)$ and scaled to unit variance $(\mathrm{SD}=1.0)$.

After determining the optimal number of factors based on the PCA and PFA results, we applied an exploratory factor analysis for that number of factors based on the Spearman correlations with imputed missing values (see section on Missing data). Afterwards, we applied a direct oblique rotation (oblimin method) to render the latent factors more interpretable. The Exploratory Factor Analysis and PFA were performed using the R package psych. 


\section{Results}

\section{Correlation matrix}

Table 2 depicts the correlation matrix based on the Spearman rank correlation test. We will only report and interpret significant correlations between different types of tests and not between subtests of a same type of test (for instance CCTT-1 vs CCTT-2, Rey 1 vs Rey-2). Such correlations are obvious, often close to one, and non-informative, as it is predictable that tests that measure closely related abilities show strong correlation.

\section{INSERT TABLE 2 ABOUT HERE}

\section{Correlations between cognitive and musical skills}

AMMA-T correlated with DSF $(r h o=0.305 ; p<0.0107)$, AMMA-R correlated with DSB $(r h o=0.246 ; p<0.0418)$. No significant correlations occurred between the AMMA and CCTT$1 \&-2$ and D2 scores.

Correlations between different general cognitive skills

DSB correlated to MR ( $r h o=0.298 ; p<0.0128)$ and also to Rey-1 $(r h o=0.316 ; p<0.0081)$.

D2 scores related to those of CCTT-2 ( $r h o=0.466 ; p<0.0001)$, as well as to Rey-2 $(r h o=0.324$; $p<0.0066)$ and Rey-3 $(r h o=0.287 ; p<0.0166)$. CCTT-2 also correlated to Rey-2 $(r h o=0.246$; $p<0.0420)$.

MR correlated to Rey-2 ( $r h o=0.296 ; p<0.0136)$ and Rey-3 (rho=0.393; $p<0.0008)$, and as already stated above, to DSB ( $r h o=0.298 ; p<0.0128)$. 


\section{Correlations between sensorimotor and musical skills}

No links were found between the different scores of the Purdue Pegboard (PP) and both musical subscores from the AMMA.

Correlations between different sensorimotor and general cognitive skills

PP-RH correlated to the CCTT-1 test $(r h o=0.287 ; p<0.017)$ and to the CCTT-2 one $(r h o=0.288 ; p<0.0163)$. The PP-RH scores also correlated to Rey-1 $(r h o=0.320 ; p<0.0074)$ and Rey-3 (rho=0.238; $p<0.0488)$.

PP-LH correlated to CCTT-2 $(r h o=0.322 ; p<0.0069)$ and to the D2 score $(r h o=0.388$; $p<0.0016)$. Then PP-LH correlated negatively to DSB $(r h o=-0.0288 ; p<0.0163)$.

The PP-Ass score correlated to MR $(r h o=0.373 ; p<0.0016)$ and to Rey-3 $(r h o=0.271$; $p<0.0241)$.

There were no relationships between AMMA-R and PP.

\section{Multivariate analyses}

Both PCA and PFA indicated an optimal number of four latent factors.

The PCA showed a clear minimum square error of prediction (MSEP) using $k$-fold cross validation (Josse \& Husson, 2012) at four components, explaining together $59.2 \%$ of the variance in the data. The PFA also indicated four factors, as the eigenvalues of the $5^{\text {th }}$ factor were larger for the random data than the ones obtained for the original data set.

In consequence, we applied an Exploratory Factor Analysis with four latent factors based on the Spearman correlations with imputed missing values (see the section on Missing Data). Ordinary least squares were used to find the minimum residual solutions. Afterwards a direct oblique rotation (oblimin method) was applied in order to make the latent factors more 
interpretable. Oblique rotation allows correlations among factors, appropriate here as certain measures are supposed to show diverse relationships.

\section{Latent factors resulting from the Exploratory Factorial Analysis}

Table 3 provides the loadings of all tests for the four factors as well as the communalities and uniqueness. Figure 1 represents the loadings of all tests on all four factors graphically. We will only interpret loadings above 0.3 .

\section{INSERT TABLE 3 ABOUT HERE}

\section{INSERT FIGURE 1 ABOUT HERE}

\section{Factor 1: "Verbal memory"}

With strong loadings for Rey-3 (0.824) \& Rey-2 (0.729) scores and moderate loadings for MR (0.485) and Rey-1 scores (0.371), this factor involves verbal memory and learning, as well as matrix reasoning.

Factor 2: "Music processing, short-term and working memory"

This factor involves musical processing with moderate loadings for the melodic $(0.455)$ and rhythmic (0.372) AMMA scores, as well as strong loadings for short-term (0.613) and working memory results $(0.707)$.

\section{Factor 3: "Manual dexterity"}

This factor involves manual dexterity and bimanual coordination, with strong loadings on this factor for PP-BH (0.754), PP-Ass (0.630) and PP-BH (0.598). The left hand (PP-LH) only 
loads at 0.211 , thus standing apart from the other three Purdue Pegboard measures. CCTT1-1 scores also load moderately on this factor (0.319).

\section{Factor 4: "Visual Processing speed and attention"}

Strong loadings by CCTT-2 (0.703) and D2 scores (0.629) and moderate loadings by CCTT-1 scores $(0.361)$ characterize this factor, which essentially involves visual processing speed and attention. The PP-LH score also saturates this factor (0.449).

\section{Discussion}

The main finding of this study is the relative idiosyncrasy of musical cognitive faculties versus higher order cognitive abilities in musically untrained children, as illustrated by their loadings on latent Factor 2: "Music processing, short-term and working memory". Relatively important loadings for Factor 2 only manifested for short-term (DSF) and working memory (DSB) and for the tonal (AMMA-T) and rhythm (AMMA-R) subscores of the "Advanced Measures of Music Audiation" (AMMA). Correlations between the AMMA scores and scores of cognition also only manifested for DSF and DSB. The latter observations partially confirm our first hypothesis (see last paragraph of the Introduction). No relationships between the AMMA scores and higher order cognitive abilities occurred. Finally, uniqueness was high for both musical subscores.

DSB apparently plays a role as a hub, also supporting other cognitive abilities. Correlations showed also with Matrix Reasoning (MR) and short-term verbal memory (Rey-1) and logically of DSF, as DSB and DSF are distinct but interdependent cognitive functions (Grégoire, 2009). Relatively high communalities for DSB support this assumption. This could explain that music practice gradually intertwines and strengthens the links between musical and cognitive skills in children. 
Finally, correlations between sensorimotor performance, measured by the different subtests of the Purdue Pegboard test, and cognitive function, supporting our second hypothesis, were quite extended. They concerned visual processing speed (CCTT-1 \& 2) and mental flexibility (CCTT-2), selective attention (D2), short term verbal memory and learning (Rey-1 and Rey-3) and matrix reasoning (MR). It seems that easily executed and automatized sensorimotor skills may allow increased neurobehavioral freedom and learning during more complex tasks (Crespo-Eguilaz et al., 2014).

\section{Correlations}

\section{Correlations between musical and other skills}

As predicted by hypothesis 1 , short-term auditory memory (DSF) and working memory (DSB) serve the detection of melodic and rhythmic differences between pairs of melodies. DSF correlated to AMMA-T, so short-term auditory memory underpinned melodic processing, allowing to retain the order of the pitches in serial order. DSB. Auditory working memory was implied in rhythmic processing, possibly reflecting involvement of the phonological loop of Baddeley's model of working memory, used to go back and forth between the first and the second melody to compare the rhythm (Baddeley, 2003). These results are also supported by the Exploratory Factor Analysis, with positive loadings on Factor 2 for both AMMA scores and both auditory memory scores (DSF \& DSB). These findings confirm earlier results of impact of working memory on music and language processing and error detection (Degé et al., 2015; James et al., 2015; Oechslin et al., 2013). We did not find any associations between visual attention, processing speed or mental flexibility, as expressed by CCTT and D2 performance, and performance on the musical tasks, infirming the second part of hypothesis 1 . Note that the findings in the literature on the association between attention, processing speed, executive function, and musical capacities are often the fruit of musical training (J. Bugos \& 
Mostafa, 2011; Bugos et al., 2007; Moreno et al., 2011; Moreno \& Farzan, 2015; Roden et al., 2014), whereas our participants were musically naïve.

Surprisingly, none of the Purdue Pegboard scores related to any of the musical scores, infirming hypothesis 3. Possibly, links between sensorimotor skills and music processing manifest only in people who practice music (Martins et al., 2018). We did not find any significant correlations between the AMMA scores and the Rey scores either. Reported relationships between verbal learning memory and musical processing skills also rather occurred in musically trained individuals (Chan et al., 1998; Roden et al., 2012).

\section{Correlations between cognitive and sensorimotor skills}

The most compelling observation was the correlation between the test of fine finger dexterity and bimanual coordination, the assembly test of the Purdue Pegboard (PP-Ass) and abstract reasoning, as measured by MR. Being able to perform complex sensorimotor skills automatically and easily, may increase neurobehavioral freedom (Crespo-Eguilaz et al., 2014). Children suffering from developmental coordination disorder who performed poorly on the Purdue Pegboard test, were also hampered for perceptual management of complex visuospatial information (Noten et al., 2014) required to well perform the MR test. PP-RH, PP-LH and PP$\mathrm{BH}$ all related to the CCTT and D2 test, showing that manual dexterity relates positively to performing these tests on visual processing speed, mental flexibility and attention. As both the CCTT and D2 are performed by hand, it seems plausible to presume that, if the procedural part of the test does require little effort, more resources are available to be mentally flexible and for rapid application of rules. Finally, at first sight less plausible findings are the correlations between PP-Ass and PP-RH with long-term verbal memory (Rey-3) and short-term verbal recall (Rey-1). This observation might be explained however by the fact that being "dexterous", 
liberates resources to focus on the outside word, resulting in enhanced capacity to retain vocabulary.

\section{Correlations between different general cognitive skills}

A series of obvious correlations occurred within the pool of cognitive skills tested here and need no extensive explanation. For instance, the association between the D2 scores and CCTT2 scores that evaluate analogous skills, like visual processing speed and attention, rule compliance and mental flexibility. Then both D2 and CCTT-2 correlated to Rey-2, D2 also to Rey-3, supporting previously observed shared variance between verbal memory and executive function (Duff et al., 2005). Another evident association linked working memory (DSB) to matrix reasoning (MR), as disclosed previously (Little et a., 2014). Links between MR and Rey-2 and Rey-3 seem less intuitive, verbal memory does not seem an ingredient of abstract thinking. But especially children may rely on semantics to perform the MR task (Mathieu, Booth, \& Prado, 2015). As could be expected also, scores of auditory working memory for numbers (DSB) and of short-term verbal memory (Rey-1) were strongly linked.

\section{Exploratory Factor Analysis}

Each of the latent factors resulting from the Exploratory Factor Analysis showed a clear fingerprint. Factor 1 was mainly saturated by the three verbal memory and learning measures (Rey-1-2-3) but most strongly by verbal learning (Rey-2-3) and less by short-term verbal memory (Rey-1). Interestingly MR also saturated this Factor, probably showing that the children depended on semantics to solve the matrix reasoning problems.

Factor 2 represents music processing and auditory short-term and working memory, confirming hypothesis 1 partially. Auditory short-term and working memory and music processing, as measured here by both subscores of the AMMA, thus share underlying processing elements 
that rely strongly on auditory recall. As no higher-order cognition tests saturated this factor, these results suggest relative independency of musical cognitive faculties in non-musician children. The high uniqueness of both AMMA-R and AMMA-T supports this supposition. At the other hand, the fact that auditory working memory also supports higher-order cognition (MR and Rey-1) suggests that musical practice might enhance the development of higher-order cognitive functioning indirectly.

Factor 3 represents sensorimotor hand and arm function as measured here by the Purdue Pegboard. CCTT-1, a simple visual processing speed task performed by hand, also relies on this latent factor. Because the CCTT-1 simply requires connecting digits in increasing order by hand on a paper sheet, this is a plausible observation.

Factor 4 embodies a latent factor representing visual processing speed and attention, grouping both CCTT and the D2 measures. Rather implausible seems the saturation by PP-LH on this factor. We suggest that visual processing speed and attention may enhance left hand function.

\section{Limitations}

The strongest limitation of this study is the relative restraint number of participants. A greater population would have been advantageous for multivariate analysis. This restriction is the consequence of a trade-off between the scope of the comprehensive behavioral testing battery and the number of children we could examine in a limited amount of time.

The adopted alpha threshold of .05 for correlations requires prudence concerning an interpretation of the results. However, the Explanatory Factor Analysis results supported the relatively weak correlational results. 


\section{Conclusion}

This study allowed to gain insight in the relative interconnection of musicality and cognition in naturally developing non-musician children. It seems that, without intensive musical practice, this interconnection is limited to basic building blocks of cognition: auditory shortterm and working memory. Links to higher-order cognition did not occur and may only manifest following protocolled music practice over longer periods of time.

A noteworthy secondary finding is the correlation of manual dexterity with higher-order cognition. Specifically, the link between bimanual coordination of fine finger dexterity, implied in the Purdue Assembly task, and abstract reasoning as measured here by the Matrices subtests of the WISC-IV, shows the importance of mastering procedural skills allowing to free resources for higher-order cognitive function. 


\section{Acknowledgements}

The authors would like to thank Mrs. Magali Peyron and Mr. Christian Forgnone, School Principals, for agreeing to conduct the study at their institutions. Special thanks go to Mrs. Magali Peyron for her persistent support to the scheduling and follow-up of the experiment. The authors are also very grateful to the children and their parents for their precious collaboration.

\section{Author Contributions}

Conceived and designed the experiment: CJ SZ MK. Performed the experiments: LA DG. Analyzed the data: CJ EDL SZ. Wrote the paper: CJ MK SZ.

\section{Funding}

This work was supported by Accademia d'Archi - Ecole de Musique (http://www.accademiaarchi.ch) with the support of CARIGEST SA in the search for anonymous sponsorship, and by the Swiss National Science Foundation (SNSF) [GZ: 100014_152841/1].

\section{ORCID}

Clara E. James

https://orcid.org/0000-0001-7480-0682

\section{Clara E. James}

Geneva School of Health Sciences HES-SO

Avenue de Champel 47

1206 Geneva

Switzerland

clara.james@hesge.ch 


\section{Tables}

\section{Table 1}

Types of tests, Measures, Acronyms and involved abilities of all items of the test battery.

\begin{tabular}{llll} 
Type of Test & Measure & Acronym & Involved ability \\
\hline Cognitive Function & & & \\
Digit span & Digit Span Forward & DSF & Short-term Memory \\
& Digit Span Backward & DSB & Working Memory \\
Test of Attention & D2 & D2 & Selective visual attention \\
Children's Color Trails Test & Subtest 1 & CCTT-1 & Visual processing speed \\
& Subtest 2 & CCTT-2 & Visual processing speed \& Mental \\
& & & flexibility \\
Matrix reasoning & & MR & $\begin{array}{l}\text { Fluid Intelligence/Abstract } \\
\text { reasoning }\end{array}$ \\
Rey Auditory Verbal Learning Test & Recall & Rey-1 & Verbal short-term memory (STM) \\
& & Rey-2 & Verbal learning
\end{tabular}

\section{$\underline{\text { Procedural Skills }}$}

Purdue Pegboard

$$
\begin{aligned}
& \text { Right Hand } \\
& \text { Left Hand } \\
& \text { Both Hands } \\
& \text { Assembly }
\end{aligned}
$$$$
\text { PP-RH }
$$$$
\text { Gross dexterity right hand }
$$$$
\text { PP-LH Gross dexterity left hand }
$$$$
\mathrm{P}-\mathrm{BH}
$$$$
\text { Bimanual coordination }
$$$$
\text { PP-Ass }
$$

Bimanual coordination \&

\section{$\underline{\text { Musicality }}$}

Advanced Measures of

Tonal

Music Audiation

Rhythmic

AMMA-T Tonal discrimination

AMMA-R Rhythmic discrimination

\section{Table 2}

Spearman rank correlations between all test measures. Rho and p-values are depicted for each test, separated by a forward slash (/) Significant correlations $(\mathrm{p}<.05)$ are highlighted in red font. $* \mathrm{p}<0.05 ; * * \mathrm{p}<0.005 ; * * * \mathrm{p}<0.0005$. For the acronyms of the tests, refer to Table 1 . 


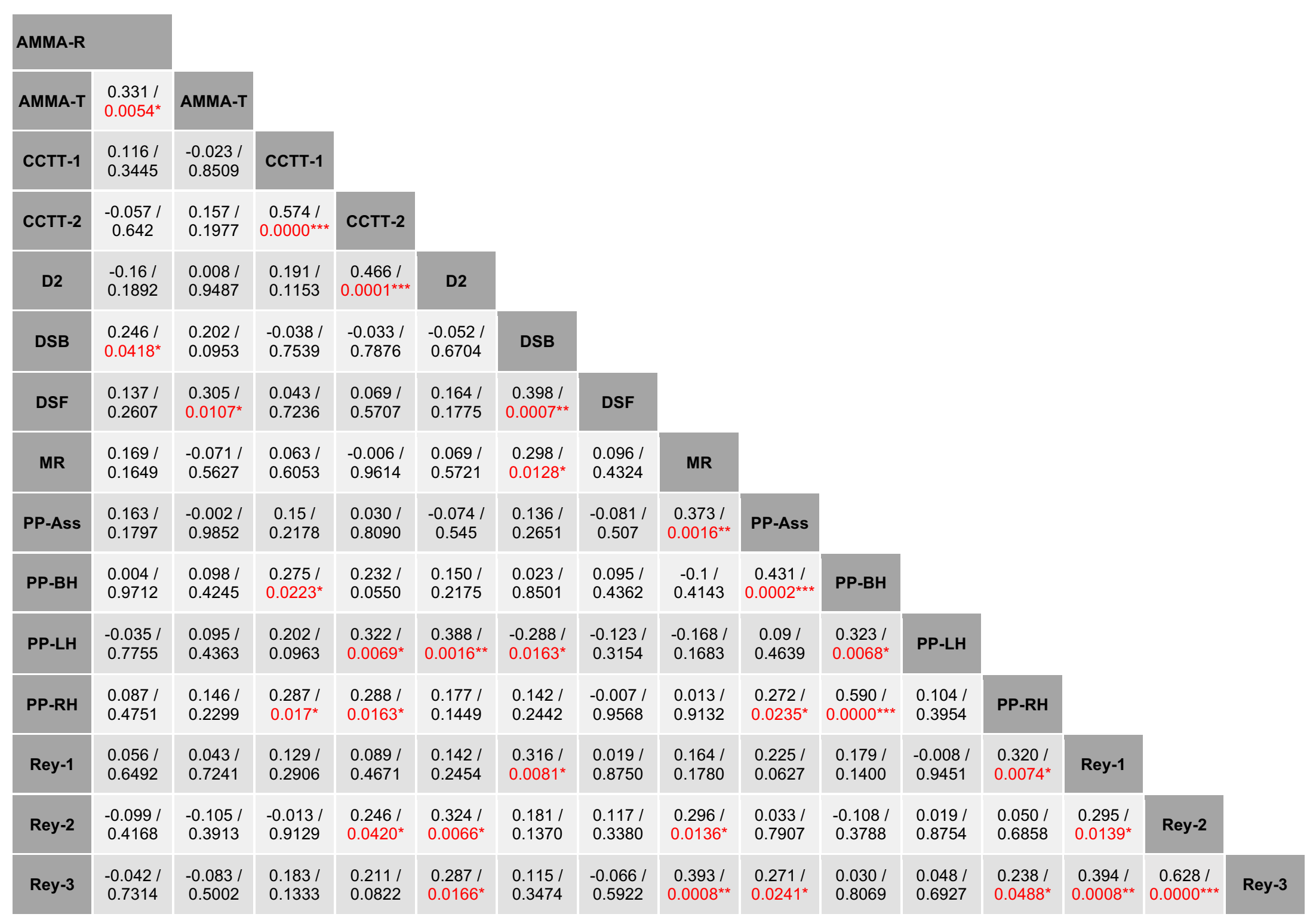




\section{Table 3}

Results of the Exploratory Factor Analysis. Loadings on the four latent factors, communalities and uniqueness are represented for each test measure.

\begin{tabular}{|l|l|l|l|l|l|l}
\hline TEST & F1 & F2 & F3 & F4 & communalities & uniqueness \\
\hline AMMA -R & -0.119 & $\mathbf{0 . 3 7 2}$ & 0.180 & -0.124 & 0.192 & 0.808 \\
\hline AMMA-T & -0.270 & $\mathbf{0 . 4 5 5}$ & 0.099 & 0.181 & 0.278 & 0.722 \\
\hline CCTT-1 & 0.048 & -0.002 & $\mathbf{0 . 3 1 9}$ & $\mathbf{0 . 3 6 1}$ & 0.273 & 0.727 \\
\hline CCTT-2 & 0.109 & 0.035 & 0.156 & $\mathbf{0 . 7 0 3}$ & 0.581 & 0.419 \\
\hline D2 & 0.253 & -0.014 & -0.047 & $\mathbf{0 . 6 2 9}$ & 0.484 & 0.516 \\
\hline DSB & 0.157 & $\mathbf{0 . 7 0 7}$ & 0.063 & -0.163 & 0.593 & 0.407 \\
\hline DSF & -0.105 & $\mathbf{0 . 6 1 3}$ & -0.112 & 0.212 & 0.400 & 0.600 \\
\hline MR & $\mathbf{0 . 4 8 5}$ & 0.195 & 0.076 & -0.224 & 0.341 & 0.659 \\
\hline PP-AsS & 0.241 & -0.011 & $\mathbf{0 . 6 3 0}$ & -0.279 & 0.499 & 0.501 \\
\hline PP-BH & -0.154 & -0.002 & $\mathbf{0 . 7 5 4}$ & 0.191 & 0.639 & 0.361 \\
\hline PP-LH & -0.068 & -0.246 & 0.211 & $\mathbf{0 . 4 4 9}$ & 0.334 & 0.666 \\
\hline PP-RH & 0.064 & 0.097 & $\mathbf{0 . 5 9 8}$ & 0.169 & 0.446 & 0.554 \\
\hline Rey-1 & $\mathbf{0 . 3 7 1}$ & 0.168 & 0.259 & -0.009 & 0.274 & 0.726 \\
\hline Rey-2 & $\mathbf{0 . 7 2 9}$ & 0.077 & -0.210 & 0.197 & 0.619 & 0.381 \\
\hline Rey-3 & $\mathbf{0 . 8 2 4}$ & -0.063 & 0.118 & 0.060 & 0.718 & 0.282 \\
\hline & & & & & & \\
\hline
\end{tabular}




\section{Figures}

\section{Figure 1}

Results of the Exploratory Factor Analysis. Color bars represent loadings for all test measures (color coding for all tests in right panel) for the four latent Factors (F1-4).

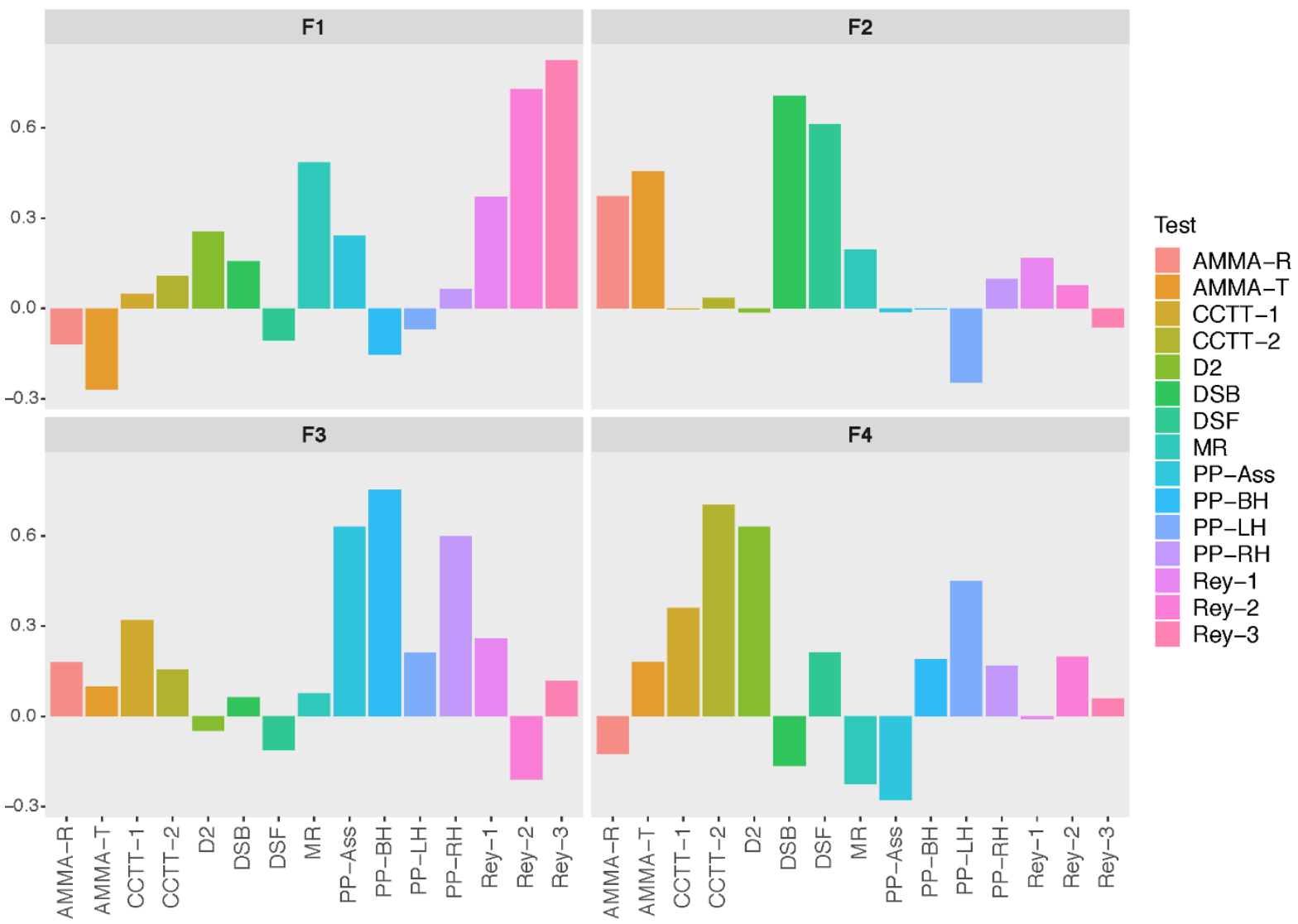




\section{Supplementary Figure 1}

Boxplots of all test scores. The boxes are arranged around the median. Dots represent outliers. For the acronyms of the tests, refer to Table 1.
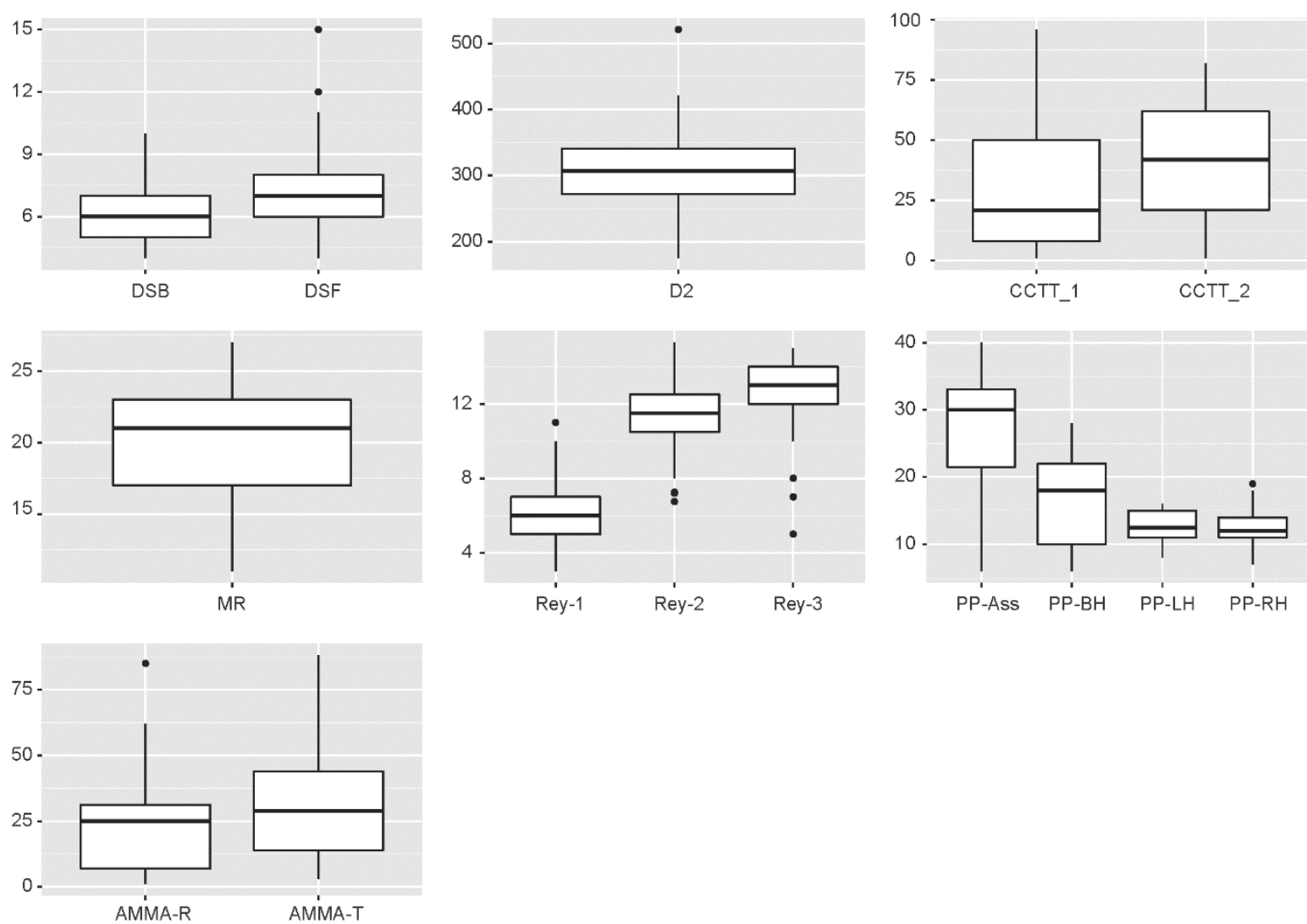


\section{References}

Anderson, D. I., Campos, J. J., Witherington, D. C., Dahl, A., Rivera, M., He, M., Uchiyama, I., \& Barbu-Roth, M. (2013). The role of locomotion in psychological development. Frontiers in Psychology, 4, Article 440. https://doi.org/10.3389/fpsyg.2013.00440

Anvari, S. H., Trainor, L. J., Woodside, J., \& Levy, B. A. (2002). Relations among musical skills, phonological processing, and early reading ability in preschool children. Journal of Experimental Child Psychology, 83(2), 111-130. http://www.ncbi.nlm.nih.gov/pubmed/12408958

Baddeley, A. (2003). Working memory: looking back and looking forward. Nature Reviews Neuroscience, 4(10), 829-839. https://doi:10.1038/nrn1201

Bean, J. (2011). Rey auditory verbal learning test, Rey AVLT. Encyclopedia of Clinical Neuropsychology, 2174-2175.

Bergman Nutley, S., Darki, F., \& Klingberg, T. (2014). Music practice is associated with development of working memory during childhood and adolescence. Frontiers in Human Neuroscience, 7, Article 926. https://doi:10.3389/fnhum.2013.00926

Brandt, A., Gebrian, M., \& Slevc, L. R. (2012). Music and early language acquisition. Frontiers in Psychology, 3, Article 327. https://doi:10.3389/fpsyg.2012.00327

Brickenkamp, R., \& Zillmer, E. (1998). The d2 Test of Attention. Hogrefe.

Bugos, J., \& Mostafa, W. (2011). Musical training enhances information processing speed. Bulletin of the Council for Research in Music Education, 7-18. https://www.jstor.org/stable/41162320

Bugos, J. A., Perlstein, W. M., McCrae, C. S., Brophy, T. S., \& Bedenbaugh, P. H. (2007). Individualized piano instruction enhances executive functioning and working memory in older adults. Aging and Mental Health, 11(4), 464-471. https://doi:10.1080/13607860601086504

Chan, A. S., Ho, Y. C., \& Cheung, M. C. (1998). Music training improves verbal memory. Nature, 396(6707), 128. https://doi:10.1038/24075

Chen, J. L., Penhune, V. B., \& Zatorre, R. J. (2008). Moving on time: brain network for auditory-motor synchronization is modulated by rhythm complexity and musical training. Journal of Cognitive Neuroscience, 20(2), 226-239. https://doi:10.1162/jocn.2008.20018

Child, D. (2006). The essentials of factor analysis: A\&C Black.

Cohrdes, C., Grolig, L., \& Schroeder, S. (2016). Relating Language and Music Skills in Young Children: A First Approach to Systemize and Compare Distinct Competencies on Different Levels. Frontiers in Psychology, 7, Article 1616. https://doi:10.3389/fpsyg.2016.01616

Courey, S. J., Balogh, E., Siker, J. R., \& Paik, J. (2012). Academic music: music instruction to engage third-grade students in learning basic fraction concepts. Educational Studies in Mathematics, 81(2), 251-278. https://doi:10.1007/s10649-012-9395-9

Crespo-Eguilaz, N., Magallon, S., \& Narbona, J. (2014). Procedural skills and neurobehavioral freedom. Frontiers in Human Neuroscience, 8, Article 449. https://doi:10.3389/fnhum.2014.00449 
Degé, F., Kubicek, C., \& Schwarzer, G. (2015). Associations between musical abilities and precursors of reading in preschool aged children. Frontiers in Psychology, 6, 12201220. https://doi:10.3389/fpsyg.2015.01220

Degé, F., Wehrum, S., Stark, R., \& Schwarzer, G. (2011). The influence of two years of school music training in secondary school on visual and auditory memory. European Journal of Developmental Psychology, 8(5), 608-623. https://doi:10.1080/17405629.2011.590668

Desjardins, C. D. a. (2018). Handbook of educational measurement and psychometrics using $R$. CRC Press.

Duff, K., Schoenberg, M. R., Scott, J. G., \& Adams, R. L. (2005). The relationship between executive functioning and verbal and visual learning and memory. Archives of Clinical Neuropsychology, 20(1), 111-122. https://doi:10.1016/j.acn.2004.03.003

Gordon, E. E. (1989). Manual for the advanced measures of muscial audiation. GIA Publications.

Gordon, R. L., Shivers, C. M., Wieland, E. A., Kotz, S. A., Yoder, P. J., \& Devin McAuley, J. (2015). Musical rhythm discrimination explains individual differences in grammar skills in children. Developmental Science, 18(4), 635-644. https://doi:10.1111/desc.12230

Grahn, J. A., \& Rowe, J. B. (2009). Feeling the beat: premotor and striatal interactions in musicians and nonmusicians during beat perception. The Journal of Neuroscience, 29(23), 7540-7548. https://doi:29/23/7540[pii]10.1523/JNEUROSCI.2018-08.2009

Grégoire, J. (2009). L'examen clinique de l'intelligence de l'enfant : fondements et pratique $d u$ WISC-IV. Mardaga.

Hannon, E. E., \& Trainor, L. J. (2007). Music acquisition: effects of enculturation and formal training on development. Trends in Cognitive Sciences, 11(11), 466-472. https://doi:10.1016/j.tics.2007.08.008

Horn, J. (1965). A rationale and test for the number of factors in factor analysis. Psychometrika, 30(2), 179-185. https://EconPapers.repec.org/RePEc:spr:psycho:v:30:y:1965:i:2:p:179-185

James, C. E., Cereghetti, D. M., Roullet Tribes, E., \& Oechslin, M. S. (2015). Electrophysiological evidence for a specific neural correlate of musical violation expectation in primary-school children. Neuroimage, 104, 386-397. https://doi:10.1016/j.neuroimage.2014.09.047

James, C. E., Dupuis-Lozeron, E., \& Hauert, C. A. (2012). Appraisal of Musical Syntax Violations by Primary School Children Effects of Age and Practice. Swiss Journal of Psychology, 71(3), 161-168. https://doi:10.1024/1421-0185/a000084

James, C. E., Michel, C. M., Britz, J., Vuilleumier, P., \& Hauert, C. A. (2012). Rhythm evokes action: early processing of metric deviances in expressive music by experts and laymen revealed by ERP source imaging. Human Brain Mapping, 33(12), 27512767. https://doi:10.1002/hbm.21397

James, C. E., Zuber, S., Dupuis-Lozeron, E., Abdili, L., Gervaise, D., \& Kliegel, M. (2020). Formal string instrument training in a class setting enhances cognitive and sensorimotor development of primary school children. Frontiers in Neuroscience, 14, Article 567. https:// 10.3389/fnins.2020.00567 
Jaschke, A. C., Eggermont, L. H., Honing, H., \& Scherder, E. J. (2013). Music education and its effect on intellectual abilities in children: a systematic review. Reviews in the Neurosciences, 24(6), 665-675. https:// 10.1515/revneuro-2013-0023

Jaschke, A. C., Honing, H., \& Scherder, E. J. A. (2018). Longitudinal Analysis of Music Education on Executive Functions in Primary School Children. Frontiers in Neuroscience, 12, Article 103. https://doi.org/10.3389/fnins.2018.00103

Josse, J., \& Husson, F. (2012). Selecting the number of components in principal component analysis using cross-validation approximations. Computational Statistics \& Data Analysis, 56(6), 1869-1879. doi:http://dx.doi.org/10.1016/j.csda.2011.11.012

Josse, J., Husson, F., \& Pagès, J. (2009). Gestion des données manquantes en analyse en composantes principales. Journal de la Société Française de Statistique, 150(2), 2851.

Karma, K. (2007). Musical Aptitude Definition and Measure Validation: Ecological Validity Can Endanger the Construct Validity of Musical Aptitude Tests. Psychomusicology, 19(2), 79-90.

Lafayette. (1999). Purdue Pegboard Model\# 32020: instructions and normative data. Lafayette Instruments.

Little, D. R., Lewandowsky, S., \& Craig, S. (2014). Working memory capacity and fluid abilities: the more difficult the item, the more more is better. Frontiers in Psychology, 5, Article 239. https://doi.org/10.3389/fpsyg.2014.00239

Llorente, A. M. (2003). Children's color trails test: professional manual. Psychological Assessment Resources.

Lorås, H., Stensdotter, A.-K., Öhberg, F., \& Sigmundsson, H. (2013). Individual Differences in Motor Timing and Its Relation to Cognitive and Fine Motor Skills. PLoS ONE, 8(7), e69353. https://doi:10.1371/journal.pone.0069353

Martins, M., Neves, L., Rodrigues, P., Vasconcelos, O., \& Castro, S. L. (2018). Orff-Based Music Training Enhances Children's Manual Dexterity and Bimanual Coordination. Frontiers in Psychology, 9(2616). https://doi:10.3389/fpsyg.2018.02616

Mathieu, R., Booth, J. R., \& Prado, J. (2015). Distributed neural representations of logical arguments in school-age children. Human Brain Mapping, 36(3), 996-1009. https://doi:10.1002/hbm.22681

Miendlarzewska, E. A., \& Trost, W. J. (2013). How musical training affects cognitive development: rhythm, reward and other modulating variables. Frontiers in Neuroscience, 7(279), 279. https://doi:10.3389/fnins.2013.00279

Moreno, S., Bialystok, E., Barac, R., Schellenberg, E. G., Cepeda, N. J., \& Chau, T. (2011). Short-term music training enhances verbal intelligence and executive function. Psychological Science, 22(11), 1425-1433. https://doi:10.1177/0956797611416999

Moreno, S., \& Farzan, F. (2015). Music training and inhibitory control: a multidimensional model. Annals of the New York Academy of Sciences, 1337, 147-152. https://doi:10.1111/nyas.12674

Moreno, S., Marques, C., Santos, A., Santos, M., Castro, S. L., \& Besson, M. (2009). Musical training influences linguistic abilities in 8-year-old children: more evidence for brain plasticity. Cerebral Cortex, 19(3), 712-723. https://doi:bhn120[pii]10.1093/cercor/bhn120 
Mous, S. E., Schoemaker, N. K., Blanken, L. M. E., Thijssen, S., van der Ende, J., Polderman, T. J. C., Jaddoe, V. W. V., Hofman, A., Verhulst, F. C., Tiemeier, H., \& White, T. (2017). The association of gender, age, and intelligence with neuropsychological functioning in young typically developing children: The Generation R study. Applied Neuropsychology: Child, 6(1), 22-40. https://doi.org/10.1080/21622965.2015.1067214

Noten, M., Wilson, P., Ruddock, S., \& Steenbergen, B. (2014). Mild impairments of motor imagery skills in children with DCD. Research in Developmental Disabilities, 35(5), 1152-1159. https://doi:10.1016/j.ridd.2014.01.026

Oechslin, M. S., Van De Ville, D., Lazeyras, F., Hauert, C. A., \& James, C. E. (2013). Degree of musical expertise modulates higher order brain functioning. Cerebral Cortex, 23(9), 2213-2224. https://doi:10.1093/cercor/bhs206

Patel, A. D. (2012). Language, music, and the brain: a resource-sharing framework. In P. Rebuschat, M. Rohrmeier, J. A. Hawkins, \& I. Cross (Eds.), Language and Music as Cognitive Systems. Oxford University Press.

Perani, D., Saccuman, M. C., Scifo, P., Spada, D., Andreolli, G., Rovelli, R., . . Koelsch, S. (2010). Functional specializations for music processing in the human newborn brain. Proceedings of the National Academy of Sciences of the United States of America,, 107(10), 4758-4763. https://doi:10.1073/pnas.0909074107

Piek, J. P., Dawson, L., Smith, L. M., \& Gasson, N. (2008). The role of early fine and gross motor development on later motor and cognitive ability. Human Movement Science, 27. https://doi:10.1016/j.humov.2007.11.002

Rauscher, F. H., Shaw, G. L., Levine, L. J., Wright, E. L., Dennis, W. R., \& Newcomb, R. L. (1997). Music training causes long-term enhancement of preschool children's spatialtemporal reasoning. Neurological Research, 19(1), 2-8.

http://www.ncbi.nlm.nih.gov/entrez/query.fcgi?cmd=Retrieve $\& d b=P u b M e d \& d o p t=C$ itation\&list uids $=9090630$

Rey, A. (1964). L'examen clinique en psychologie [The clinical psychological examination]. Presses Universitaires de France.

Rickard, N. S., Bambrick, C. J., \& Gill, A. (2012). Absence of widespread psychosocial and cognitive effects of school-based music instruction in 10-13-year-old students. International Journal of Music Education, 30(1), 57-78. https://doi:10.1177/0255761411431399

Roden, I., Könen, T., Bongard, S., Frankenberg, E., Friedrich, E., \& Kreutz, G. (2014). Effects of Music Training on Attention, Processing Speed and Cognitive Music Abilities-Findings from a Longitudinal Study. Applied Cognitive Psychology, 28(4), 545-557. https://doi:10.1002/acp.3034

Roden, I., Kreutz, G., \& Bongard, S. (2012). Effects of a school-based instrumental music program on verbal and visual memory in primary school children: a longitudinal study. Frontiers in Psychology, 3, Article 572. https://doi:10.3389/fpsyg.2012.00572

Rose, D. C., Jones Bartoli, A., \& Heaton, P. (2015). A study of cognitive and behavioural transfer effects associated with children learning to play musical instruments for the first time over one academic year. The Psychology of Education Review. https://uhra.herts.ac.uk/handle/2299/18872

Schellenberg, E. G. (2004). Music lessons enhance IQ. Psychological Science, 15(8), 511514. https://doi:10.1111/j.0956-7976.2004.00711.x 
Schellenberg, E. G. (2006). Long-term positive associations between music lessons and IQ. Journal of educational psychology, 98(2), 457-468. https://doi:10.1037/00220663.98.2.457

Seither-Preisler, A., Parncutt, R., \& Schneider, P. (2014). Size and synchronization of auditory cortex promotes musical, literacy, and attentional skills in children. The Journal of Neuroscience, 34(33), 10937-10949. https://doi:10.1523/JNEUROSCI.5315-13.2014

Suggate, S., \& Stoeger, H. (2017). Fine motor skills enhance lexical processing of embodied vocabulary: A test of the nimble-hands, nimble-minds hypothesis. The Quarterly Journal of Experimental Psychology, 70(10), 2169-2187. https://doi:10.1080/17470218.2016.1227344

Wang, M. V., Lekhal, R., Aaro, L. E., Holte, A., \& Schjolberg, S. (2014). The developmental relationship between language and motor performance from 3 to 5 years of age: a prospective longitudinal population study. BMC Psychology, 2(1), 34. https://doi:10.1186/s40359-014-0034-3

Wechsler, D. (2003). Wechsler intelligence scale for children, 4th edition (WISC-IV®). The Psychological Corporation.

Wechsler, D. (2005). WISC-IV : échelle d'intelligence de Wechsler pour enfants et adolescents. [Wechsler Intelligence Scale for Children and Adolescents]. ECPA.

Winkler, I., Haden, G. P., Ladinig, O., Sziller, I., \& Honing, H. (2009). Newborn infants detect the beat in music. Proceedings of the National Academy of Science USA, 106(7), 2468-2471. https://doi:0809035106[pii]10.1073/pnas.0809035106 